\title{
DESFECHOS DE TRANSPLANTES RENAIS COM RINS PAREADOS
}

\author{
Outcomes of the paired kidney transplantation
}

\begin{abstract}
Ana Carine Goersch Silva',2, Clarissa Ferreira Lobo, ${ }^{1,5}$, Gilberto Loiola de Alencar Dantas ${ }^{4}$, Rita Mônica Borges Studart',2, Juliana Gomes Ramalho de Oliveira ${ }^{3}$, Celi Melo Girão ${ }^{1}$, Tainá Veras de Sandes-Freitas ${ }^{1,4,5}$ Ronaldo de Matos Esmeraldo
\end{abstract}

\section{RESUMO}

Objetivo: Avaliar os desfechos do transplante renal (TxR) em receptores de rins pareados e analisar os fatores de risco para desfechos inferiores. Métodos: Estudo retrospectivo de centro único incluindo todos os TxR com doador falecido realizados em 2014 em que ambos os rins foram transplantados em receptores do nosso centro. Os pacientes foram divididos em receptores do rim direito ou esquerdo. Foram avaliados os seguintes desfechos: função tardia do enxerto (FTE) rejeição aguda, perda, óbito, taxa de filtração glomerular estimada (TFGe) e incidência do desfecho composto de perda, óbito ou TFG $<50 \mathrm{~mL} / \mathrm{min}$ em dois anos. A análise de risco foi feita através de regressão logística binária. Resultados: Os doadores eram predominantemente jovens ( $29 \pm 14$ anos), homens ( $71 \%)$, que faleceram por trauma craniano (70\%). Apenas dois doadores (6\%) eram de critério expandido. Os receptores foram similares quanto à demografia: homens (77 vs. $57 \%, p=0,126)$, jovens $(35 \pm 20$ vs. $37 \pm 17$ anos, $p=0,668)$, com doença renal crônica de etiologia indeterminada ( 31 vs. $40 \%, p=0,179)$, estando em média $31 \pm 37$ meses em diálise $(27 \pm 25$ vs. $36 \pm 47$ meses, $\mathrm{p}=0,311), 7 \%$ eram retransplantes ( 9 vs. $5 \%, p=0,309)$ e as médias de PRA de classe I e II foram $6 \pm 20 \%(9 \pm 23$ vs. $10 \pm 19 \%, p=0,907)$ e $9 \pm 20 \%$ ( $9 \pm 25$ vs. $5 \pm 15 \%, p=0,476)$, respectivamente. O tempo médio de isquemia fria foi de $25 \pm 9$ h ( $24 \pm 9$ vs. $27 \pm 9 h, p=0,269)$ e $66 \%$ (66 vs. $66 \%, p=1,000)$ foram perfundidos em máquina de perfusão pulsátil. Todos foram induzidos com globulina anti-timócito, $52 \%$ (54 vs. 49\%, $p=0,808)$ receberam tacrolimo e everolimo como regime imunossupressor inicial e $62 \%$ (69 vs. $54 \%, p=0,319)$ não receberam corticoide. Não houve diferença entre os grupos quanto à incidência de FTE (20 vs. $32 \%, p=0,282)$, rejeição aguda $(0$ vs. $11 \%, p=0,054)$, perda $(0$ vs. $6 \%, p=0,493)$, óbito ( 6 vs. $6 \%, p=1,000)$, TFGe $(71 \pm 28$ vs. $72 \pm 37 \mathrm{~mL} / \mathrm{min}, p=0,877)$ ou quanto à incidência do desfecho composto de perda, óbito ou TFGe $<50 \mathrm{~mL} / \mathrm{min}$ em 2 anos $(29$ vs. $23 \%, p=0,785)$. Em análise multivariada, o único fator de risco para o desfecho composto foi FTE (OR 4,120, IC 95\% 1,194-14,271, p=0,025). Conclusão: Nesta coorte de TxR com rins pareados, FTE foi o único fator de risco para desfechos inferiores.

Descritores: Transplante de Rim; Aloenxertos; Medidas de associação risco ou desfecho; Função Retardada do Enxerto.

\section{Instituições:}

${ }^{1}$ Setor de Transplantes, Hosp.Geral de Fortaleza, Fortaleza/CE

${ }^{2}$ Mestrado Profissional em Tecnologia e Inovação em

Enfermagem, Universidade de Fortaleza (UNIFOR), Fortaleza/CE

${ }^{3}$ Programa de Pós-Graduação em Saúde Coletiva, Universidade de Fortaleza (UNIFOR), Fortaleza/CE

${ }^{4}$ Departamento de Medicina Clínica (DMC), Universidade Federal do Ceará (UFC), Fortaleza/CE

${ }^{5}$ Mestrado Profissional em Transplantes, Universidade Estadual do Ceará (UECE), Fortaleza/CE

\section{Correspondência:}

Ana Carine Goersch Silva

Hospital Geral de Fortaleza - Setor de Transplantes ( $1^{\circ}$ andar)

End.: Rua Avila Goulart, 900, CEP 60175-295, Fortaleza-CE

E-mail: carinegoersch@hotmail.com

Tel.: (85) 3242.6742

\section{INTRODUÇÃO}

O transplante renal é a terapia de escolha para doença renal crônica (DRC) em estádio terminal por proporcionar ao paciente menor morbimortalidade e melhor qualidade de vida. ${ }^{1}$ Os desfechos de um transplante dependem de eventos relacionados à seleção de doadores, preservação do enxerto, implantação cirúrgica, tratamento imunossupressor e características do receptor. ${ }^{2,3}$ 
O rim esquerdo possui uma veia renal mais longa que o rim direito, o que facilita o ato cirúrgico do implante, potencialmente reduzindo o tempo cirúrgico e as complicações técnicas e clínicas consequentes. Os estudos disponíveis comparando os desfechos do transplante entre receptores de rins direito e esquerdo têm resultados conflitantes, mas a maioria aponta para a ausência de impacto significativo da lateralidade sobre os desfechos, especialmente os desfechos duros de sobrevida do enxerto, sobrevida do paciente ou função renal. ${ }^{4-9}$

O objetivo deste estudo foi descrever os desfechos do transplante renal (FTE, sobrevidas e função renal) de rins pareados em uma coorte de transplantes renais realizados em um serviço onde predominam os transplantes com doadores falecidos de critério padrão, ou seja, doadores jovens, sem antecedentes de hipertensão arterial, com morte traumática e sem comprometimento da função renal. Foram avaliados ainda os fatores de risco para desfechos inferiores nesta coorte.

\section{MÉTODOS}

Trata-se de um estudo retrospectivo de centro único, realizado em unidade de transplante renal de Hospital Público Terciário do município de Fortaleza, Ceará, Brasil, no período de janeiro a dezembro de 2014.

A amostra foi composta por 35 doadores e 70 receptores. Foram incluídos todos os receptores de transplante renal e seus respectivos doadores falecidos realizados em 2014, em que ambos os rins foram transplantados no mesmo serviço. Os receptores foram divididos em dois grupos, conforme a lateralidade do rim transplantado, grupo E: pacientes que receberam o rim esquerdo e grupo D: pacientes que receberam o rim direito. O termo "pares de rim" referiu-se aos indivíduos que receberam rins do mesmo doador falecido.

As seguintes variáveis do doador foram avaliadas: sexo, idade, causa do óbito, creatinina final, classificação UNOS quanto ao tipo de doador (expandido ou padrão). Dos receptores, as seguintes variáveis foram incluídas: idade, sexo, etiologia da DRC, tempo em diálise, retransplante, PRA. As variáveis relacionadas ao transplante foram: tipo de perfusão, tempo de isquemia fria e imunossupressão inicial. As variáveis de desfecho foram: incidência de FTE, número de sessões de hemodiálise após o transplante, período de internação hospitalar, rejeição aguda, perda do enxerto, óbito, função renal em dois anos e desfecho composto de perda, óbito ou TFGe $<50 \mathrm{~mL} / \mathrm{min}$ em dois anos.
De acordo com o protocolo do serviço, são submetidos à perfusão dinâmica em máquina de perfusão rins que apresentem um dos critérios a seguir: doadores acima de 50 anos, aqueles com creatinina sérica final acima de $1,5 \mathrm{mg} / \mathrm{dL}$ ou aqueles cujo tempo de isquemia fria prevista é superior a $20 \mathrm{~h}$.

Foram considerados doadores de critério expandido (DCE) aqueles com idade superior a 60 anos (ou entre 50 e 59) e que possuíssem dois dos seguintes fatores: história de hipertensão, óbito por acidente vascular encefálico e creatinina superior a $1,5 \mathrm{ml} / \mathrm{dL}$ na captação. ${ }^{10}$

A ocorrência de FTE foi considerada como a necessidade de diálise na primeira semana após o transplante. ${ }^{11}$

Para cálculo da TFGe em dois anos, utilizou-se a fórmula Modification of Diet in Renal Disease (MDRD) de quatro variáveis, ${ }^{12}$ realizando o seguinte ajuste: para as perdas do enxerto foi atribuído valor de $0 \mathrm{~mL} / \mathrm{min}$; para os óbitos e perdas de seguimento, a última creatinina disponível foi considerada para o cálculo (last observation carried forward adjustment). ${ }^{13}$

Rejeição aguda foi definida de acordo com a classificação de Banff $2013^{14}$ e perda do enxerto foi definido como o retorno definitivo para diálise.

Os dados foram coletados a partir dos formulários de acompanhamento pós-transplante e transcritos para um banco de dados no programa Microsoft Excel®. As análises estatísticas foram realizadas por meio do programa SPSS, versão 20. As variáveis categóricas foram descritas por meio de frequências e porcentagem e as numéricas por meio de média \pm desvio padrão. $\mathrm{O}$ teste Kolmogorov-Smirnov foi utilizado para verificar a normalidade de distribuição das variáveis e o teste de Levene para comparação da variabilidade. Nas variáveis com distribuição normal, utilizou-se o teste t de Student, para a comparação de médias. Na análise de associação entre as variáveis do estudo foram usados os testes de Pearson, qui-quadrado e Fisher. Para análise multivariada foi utilizada regressão logística binária stepwise forward. As variáveis com valor de $p$ inferior a $1 \%$ na análise univariada foram incluídas no modelo. Para a análise com múltiplas variáveis, o valor de $p<0,05$ e o intervalo de confiança (IC) 95\% excluindo a unidade foram utilizados para considerar a significância estatística. Para as demais análises comparativas, o valor de $\mathrm{p}$ foi considerado significante quando inferior a $5 \%$.

Este estudo foi realizado seguindo os preceitos éticos preconizados pela resolução 466/12 do Conselho Nacional de Saúde do Ministério da Saúde e aprovado pelo Comitê de Ética em Pesquisa do Hospital Geral de Fortaleza (CAEE 34237914.2.0000.5040). 
Ana Carine Goersch Silva, Clarissa Ferreira Lobo, Gilberto Loiola de Alencar Dantas, Rita Mônica Borges Studart, Juliana Gomes Ramalho de Oliveira, Celi Melo Girão, Tainá Veras de Sandes-Freitas, Ronaldo de Matos Esmeraldo

\section{RESULTADO}

O grupo dos doadores foi predominantemente composto por adultos jovens ( $29 \pm 14$ anos), do sexo masculino (71\%), tendo como principal causa de óbito traumatismo crânio encefálico (TCE) $(60 \%)$, com creatinina final média de $1,4 \pm 0,9 \mathrm{mg} / \mathrm{dL}$ e apenas $6 \%$ eram DCE (Tabela 1 ).

As características demográficas dos receptores foram semelhantes entre os grupos. Os receptores dessa amostra eram predominantemente adultos jovens $(35 \pm 20$ vs. $37 \pm 17$ anos, $p=0,668$ ), do sexo masculino (77 vs. $57 \%, p=0,126)$, portadores de doença renal crônica de causa indeterminada (31 vs. $40 \%, p=0,179$ ). Eram predominantemente pacientes de baixo risco imunológico. Todos foram induzidos com anticorpo depletor de linfócitos (Thymoglobuline ${ }^{\circledR}$ ) e a maioria recebeu como regime imunossupressor inicial a combinação de tacrolimo e everolimo (54 vs. $49 \%, p=0,808$ ), sem corticoide (69 vs. $54 \%, p=0,319)$ (Tabela 2).

Não houve diferença estatisticamente significante entre os grupos quanto à incidência de FTE (20 vs. $32 \%$, $p=0,282$ ), quanto ao número de sessões de hemodiálise após o transplante $(4 \pm 2$ vs. $3 \pm 3, p=0,922)$ e período de internação hospitalar ( $14 \pm 9$ vs. $15 \pm 10, p=0,576)$. Não houve casos de rejeição aguda e perda do enxerto no grupo $E$, enquanto no grupo $D$ ocorreram quatro casos de rejeição aguda (11\%) e dois casos de perda do enxerto (6\%). A incidência de óbitos foi semelhante entre os grupos ( 6 vs. $6 \%, p=1,000$ ) e não foi constatada diferença quanto à função renal em dois anos $(71 \pm 28$ vs. e $72 \pm 37 \mathrm{~mL} / \mathrm{min}, p=0,877$ ). Quanto ao desfecho composto de perda, óbito ou TFGe $<50 \mathrm{~mL} / \mathrm{min}$ em dois anos, os grupos também foram semelhantes (29 vs. $23 \%, p=0,785$ ) (Tabela 3).

Em análise univariada, a idade do receptor e a ocorrência de FTE foram fatores de risco para o desfecho composto. $\mathrm{Na}$ análise multivariada, apenas FTE foi identificada como fator de risco independente (OR 4,120, IC 95\% 1,194-14,271, p=0,025) (Tabela 4).

Tabela 1: Caracterização dos doadores falecidos no ano de 2014. Fortaleza, Ceará.

\begin{tabular}{lc}
\hline \multicolumn{1}{c}{ Variáveis } & $\mathbf{N}=\mathbf{3 5}$ \\
\hline Sexo, $\mathrm{n}(\%)$ & $25(71)$ \\
Masculino & $29 \pm 14$ \\
Idade em anos (média $\pm \mathrm{DP})$ & \\
Causa do óbito, $\mathrm{n}(\%)$ & $21(60)$ \\
TCE & $10(29)$ \\
AVC & $4(11)$ \\
Outra & $1,4 \pm 0,9$ \\
Creatinina final (mg/dL) (média $\pm \mathrm{DP})$ & $2(6)$ \\
DCE, $\mathrm{n}(\%)$ & \\
\hline
\end{tabular}

TCE: trauma crânio encefálico; AVC: acidente vascular cerebral; DCE: doador de critério expandido; DP: desvio padrão
Tabela 2: Caracterização dos receptores no ano de 2014, Fortaleza, Ceará

\begin{tabular}{|c|c|c|c|}
\hline Variável & $\begin{array}{c}\text { Grupo } E \\
N=35\end{array}$ & $\begin{array}{c}\text { Grupo D } \\
\mathrm{N}=35\end{array}$ & $\begin{array}{c}p \\
\text { valor }\end{array}$ \\
\hline Idade em anos (média \pm DP) & $35 \pm 20$ & $37 \pm 17$ & 0,668 \\
\hline Sexo masculino, $\mathrm{n}(\%)$ & $27(77)$ & $20(57)$ & 0,126 \\
\hline $\begin{array}{l}\text { Etiologia da DRC } \\
\text { Indeterminada } \\
\text { HAS } \\
\text { DM } \\
\text { GNC } \\
\text { Outros }\end{array}$ & $\begin{array}{c}11(31) \\
5(14) \\
5(14) \\
4(11) \\
10(29)\end{array}$ & $\begin{array}{l}14(40) \\
1(3) \\
2(6) \\
9(26) \\
9(26)\end{array}$ & \\
\hline Tempo em diálise (meses) (média \pm DP) & $27 \pm 25$ & $36 \pm 47$ & 0,311 \\
\hline Retransplante, n (\%) & $3(9)$ & $2(6)$ & 0,309 \\
\hline PRA I (\%) (média \pm DP) & $9 \pm 23$ & $10 \pm 19$ & 0,807 \\
\hline PRA II (\%) (média \pm DP) & $9 \pm 25$ & $5 \pm 15$ & 0,476 \\
\hline Perfusão em MPP, n(\%) & $23(66)$ & $23(66)$ & 1,000 \\
\hline TIF (h) (média \pm DP) & $24 \pm 9$ & $27 \pm 9$ & 0,269 \\
\hline $\begin{array}{l}\text { IMS inicial, } \mathrm{n}(\%) \\
\text { TAC+EVR } \\
\text { TAC+MPS }\end{array}$ & $\begin{array}{l}19(54) \\
16(49)\end{array}$ & $\begin{array}{l}16(49) \\
19(54)\end{array}$ & 0,808 \\
\hline Regime inicial livre de esteroides, $n$ (\%) & $24(69)$ & $20(54)$ & 0,319 \\
\hline
\end{tabular}

DRC: doença renal crônica; HAS: hipertensão arterial sistêmica; DM: diabetes melito; GNC: glomerulonefrite crônica; PRA: painel de reatividade de anticorpos anti-HLA; IMS: imunossupressão; TAC: tacrolimo; EVR: everolimo; TIF: tempo de isquemia fria; MPP: máquina de perfusão pulsátil; DP: desvio padrão

Tabela 3: Desfechos dos Grupos E e D após o transplante renal. Fortaleza, Ceará, 2014.

\begin{tabular}{|c|c|c|c|}
\hline Variável & $\begin{array}{l}\text { Grupo E } \\
(\mathrm{N}=35)\end{array}$ & $\begin{array}{c}\text { Grupo D } \\
(\mathrm{N}=35)\end{array}$ & $p$ valor \\
\hline FTE, n (\%) & $7(20)$ & $11(32)$ & 0,282 \\
\hline Sessões de HD, (média $\pm D P$ ) & $4 \pm 2$ & $3 \pm 3$ & 0,922 \\
\hline Tempo de internação (dias), (média \pm DP) & $14 \pm 9$ & $15 \pm 10$ & 0,576 \\
\hline Rejeição aguda, n (\%) & $0(0)$ & $4(11)$ & 0,054 \\
\hline \multicolumn{4}{|l|}{ Perda do enxerto, $n(\%)$} \\
\hline IFTA não imune & 0 & 1 & \\
\hline Trombose vascular & 0 & 1 & \\
\hline Óbito, n (\%) & $2(6)$ & $2(6)$ & 1,000 \\
\hline Choque hemorrágico & 0 & 1 & \\
\hline Cardiovascular & 1 & 1 & \\
\hline Causa desconhecida & 1 & 0 & \\
\hline TFGe em 2 anos (mL/min), (média \pm DP) & $71 \pm 28$ & $72 \pm 37$ & 0,877 \\
\hline Desfecho composto, n (\%) & $10(29)$ & $8(16)$ & 0,785 \\
\hline
\end{tabular}

FTE: função tardia do enxerto; HD: hemodiálise; IFTA: Fibrose Intersticial e atrofia tubular; TFGe: taxa de filtração glomerular estimada; DP: desvio padrão

\section{DISCUSSÃO}

Os dados deste estudo demonstraram que a lateralidade do rim não impactou nos desfechos do transplante e que a ocorrência de FTE foi o único fator de risco independente para desfechos inferiores. 
Desfechos de transplantes renais com rins pareados

Tabela 4: Fatores de risco para o desfecho composto de perda, óbito ou TFGe $<50 \mathrm{~mL} / \mathrm{min}$ em dois anos. Fortaleza, Ceará

\begin{tabular}{|c|c|c|}
\hline & Análise univariada & Análise multivariada \\
\hline Idade do receptor (anos) & $\begin{array}{c}\text { OR 1,035, IC 95\% 1,002-1,071 } \\
p=0,040\end{array}$ & $\begin{array}{c}\text { OR 0,783, IC 95\% 0,945-1,043 } \\
p=0,783\end{array}$ \\
\hline Sexo masculino & $\begin{array}{c}\text { OR 0,496, IC 95\% 0,143-1,726 } \\
\text { p=0,271 }\end{array}$ & - \\
\hline Tempo em diálise (meses) & $\begin{array}{c}\text { OR 1,009, IC 95\%0,996-1,022 } \\
p=0,193\end{array}$ & - \\
\hline Retransplante & $\begin{array}{l}\text { OR 1,695, IC 95\% 0,487-5,898 } \\
\qquad=0,407\end{array}$ & - \\
\hline PRA I (\%) & $\begin{array}{c}\text { OR 0,987, IC 95\% 0,955-1,022 } \\
\text { p=0,421 }\end{array}$ & - \\
\hline PRA II (\%) & $\begin{array}{c}\text { OR 0,998, IC 95\% 0,971-1,025 } \\
\text { p=0,870 }\end{array}$ & - \\
\hline IMS inicial - TAC-EVR & $\begin{array}{c}\text { OR 1,080, IC 95\% 0,352-3,313 } \\
p=0,893\end{array}$ & - \\
\hline Prednisona & $\begin{array}{c}\text { OR 0,960, IC 95\% 0,302-3,050 } \\
p=0,945\end{array}$ & - \\
\hline TIF (h) & $\begin{array}{c}\text { OR 1,009, IC 95\% 0,950-1,072 } \\
\text { p=0,771 }\end{array}$ & - \\
\hline Máquina de perfusão & $\begin{array}{c}\text { OR 1,059, IC 95\% 0,341-3,292 } \\
p=0,921\end{array}$ & - \\
\hline Lateralidade do rim transplantado & $\begin{array}{c}\text { OR 0,741, IC } 95 \% 0,252-2,175 \\
\qquad P p=0,585\end{array}$ & - \\
\hline FTE & $\begin{array}{c}\text { OR 5,375, IC 95\% 1,630-17,721 } \\
p=0,006\end{array}$ & $\begin{array}{c}\text { OR 4,120, IC 95\% 1,194-14,271 } \\
p=0,025\end{array}$ \\
\hline Rejeição aguda & $\begin{array}{c}\text { OR 0,674, IC 95\% 0,185-0,245 } \\
p=0,549\end{array}$ & - \\
\hline
\end{tabular}

PRA: painel de reatividade de anticorpos; IMS: imunossupressão; TAC: tacrolimo; EVR: everolimo; TIF: tempo de isquemia fria;

FTE: função tardia do enxerto; OR: odds ratio; IC: intervalo de confiança.

Apesar das características anatômicas do rim esquerdo serem habitualmente mais favoráveis ao transplante que o rim direito, este estudo e estudos realizados anteriormente não demonstraram impacto da lateralidade nos desfechos. ${ }^{5,6}$ Estudo prévio em que também foram analisados os pares de rim, não houve diferença significativa da evolução entre os grupos, quando avaliado a TFGe, estimada usando o MDRD. ${ }^{7}$

Convém ressaltar que a população dessa coorte foi composta predominantemente por um binômio doadorreceptor bastante favorável e peculiar da região onde o estudo foi realizado: receptores jovens e de baixo risco imunológico e, mais importante, doadores jovens, os quais morreram por trauma craniano. Além disso, $60 \%$ dos rins foram perfundidos em máquina de perfusão pulsátil após um período de preservação estática. Apesar dessas características demográficas bastante favoráveis, a incidência de FTE foi significativamente elevada e impactou nos desfechos. Enquanto estudos com coortes de transplantes americanas e europeias reportam incidências de FTE da ordem de $20-25 \%$ e $30 \%$, respectivamente, a incidência de FTE nos centros brasileiros varia entre $50-70 \% .{ }^{15}$ Apesar de bem abaixo da incidência observada na população brasileira, a incidência de DGF estimada para essa população está abaixo de $20 \%$, considerando o perfil demográfico da combinação doador-receptor da coorte. ${ }^{16} \mathrm{~A}$ manutenção hemodinâmica inadequada do doador está implicada como uma possível causa. ${ }^{15}$

É conhecido o impacto da FTE nos desfechos do transplante renal. ${ }^{17}$ Evidências apontam um grau de correlação entre a ocorrência de FTE e a sobrevida dos enxertos quando comparados os resultados do transplante renal entre pares de receptores.

Segundo Louvar et al (2009), quando um receptor apresentou FTE, o receptor do rim contralateral apresentou maior risco para desenvolvimento da mesma complicação. Pesquisadores demonstraram que a ocorrência de FTE, rejeição aguda e falência do enxerto estão associadas ao aumento do tempo de permanência hospitalar e dos custos do tratamento. ${ }^{4}$ As diferenças numéricas observadas na incidência de rejeição aguda e perda do enxerto não foram estatisticamente significativas e, portanto, consideradas sem relevância.

Este estudo tem limitações importantes, as quais devem ser apontadas: trata-se de um estudo de centro único com amostra limitada e número reduzido de desfechos. 
Além disso, a natureza retrospectiva impediu a obtenção fidedigna de informações que dependam de registro nos prontuários. No entanto, desconhecemos estudos brasileiros avaliando os desfechos de pares de rim, especialmente em uma coorte com características demográficas e clínicas tão peculiares.

\section{CONCLUSÃO}

Em conclusão, a lateralidade do rim transplantado não se associou à perda, ao óbito ou à função renal inferior. Função tardia do enxerto foi prevalente e associada independentemente ao desfecho composto.

\section{ABSTRACT}

Purpose: We aimed to assess kidney transplant (KT) outcomes in paired kidney recipients and analyzing the risk factors for inferior outcomes. Methods: single center retrospective study including all deceased donor KT performed in 2014 in which both kidneys were transplanted into our patients. Patients were divided in two groups according to the laterality of the transplanted kidney. The following outcomes were assessed: delayed graft function (DGF), acute rejection, graft loss, death, glomerular filtration rate (GFR) and incidence of the composite endpoint of loss, death or GFR $<50 \mathrm{~mL} / \mathrm{min}$ at 2 years. Risk analysis was performed by using binary logistic regression. Results: Donors were predominantly young $(29 \pm 14$ years) men $(71 \%)$ who died because of head trauma $(70 \%)$. Only $2(6 \%)$ were expanded criteria donors. Demography of KT recipients were similar between groups: men ( 77 vs. $57 \%, p=0.126)$, young ( $35 \pm 20$ vs. $37 \pm 17$ years old, $p=0.668$ ), with unknown etiology for chronic kidney disease (31 vs. $40 \%, p=0.179$ ), who were $31 \pm 37$ months $(27 \pm 25$ vs. $36 \pm 47$ months, $p=0.311)$ on dialysis before $K T$. $7 \%$ were retransplants $(9$ vs. $5 \%, p=0.309)$ and Class I and II PRA were $6 \pm 20 \%(9 \pm 23$ vs. $10 \pm 19 \%, p=0.907)$ and $9 \pm 20 \%(9 \pm 25$ vs. $5 \pm 15 \%, p=0.476)$, respectively. The mean cold ischemia time was $25 \pm 9$ hours $(24 \pm 9$ vs. $27 \pm 9$ hours, $p=0.269)$ and $66 \%(66$ vs. $66 \%, p=1.000)$ were pumped into a pulsatile perfusion machine. All patients were induced with antithymocyte globulin, $52 \%(54$ vs. $49 \%$, $\mathrm{p}=0.808)$ received tacrolimus and everolimus as initial immunosuppressive regimen, and $62 \%$ were steroid-free $(69$ vs. $54 \%, p=0.319)$. There were no differences between groups on the incidence of DGF (20 vs. $32 \%, p=0.282)$, acute rejection ( 0 vs. $11 \%, p=0.054)$, graft loss ( 0 vs. $6 \%, p=0.493)$, death $(6$ vs. $6 \%, p=1.000)$, GFR $(71 \pm 28$ vs. $72 \pm 37 \mathrm{~mL} /$ $\min , p=0.877$ ) or on the incidence of the composite outcome of graft loss, death or GFR $<50 \mathrm{~mL} / \mathrm{min}$ at 2 years (29 vs. $23 \%, p=0.785)$. In multivariate analysis, DGF was the variable independently associated to the composite outcome (OR 4.120, 95\% Cl 1.194-14.271, $p=0.025)$. Conclusion: In this cohort of paired kidney transplants with optimum donors, DGF was the only risk factor for inferior outcomes.

Keywords: Kidney Transplant; Allografts; Measures of association, exposure, risk or outcome; Delayed Graft Function.

\section{REFERÊNCIAS}

1. Tonelli M, Wiebe N, Knoll G, Bello A, Browne S, Jadhav D, et al. Systematic review: kidney transplantation compared with dialysis in clinically relevant outcomes. Am J Transplant. 2011;11(10):2093-109.

2. Harada KM, Mandia-Sampaio EL, de Sandes-Freitas TV, Felipe CR, Park SI, Pinheiro-Machado PG, et al. Risk factors associated with graft loss and patient survival after kidney transplantation. Transplant Proc. 2009;41(9):3667-70.

3. Kim SJ, Lee HH, Lee DS, Lee KW, Joh JW, Woo DH, et al. Prognostic factors affecting graft and patient survival in cadaveric and living kidney transplantation. Transplant Proc. 2004;36(7):2038-9.
4. Louvar DW, Li N, Snyder J, Peng Y, Kasiske BL, Israni AK. "Nature versus nurture" study of deceased-donor pairs in kidney transplantation. J Am Soc Nephrol. 2009;20(6):1351-8.

5. Phelan PJ, Shields W, O'Kelly P, Pendergrass M, Holian J, Walshe JJ, et al. Left versus right deceased donor renal allograft outcome. Transpl Int. 2009;22(12):1159-63.

6. Johnson DW, Mudge DW, Kaisar MO, Campbell SB, Hawley $\mathrm{CM}$, Isbel NM, et al. Deceased donor renal transplantation-does side matter? Nephrol Dial Transplant. 2006;21(9):2583-8.

7. Johnson JF, Jevnikar AM, Mahon JL, Muirhead N, House AA. Fate of the mate: the influence of delayed graft function in renal transplantation on the mate recipient. Am J Transplant. 2009;9(8):1796-801. 
8. Traynor C, O'Kelly P, Denton M, Magee C, Conlon PJ. Concordance of outcomes of pairs of kidneys transplanted into different recipients. Transpl Int. 2012;25(9):918-24.

9. Doshi MD, Garg N, Reese PP, Parikh CR. Recipient risk factors associated with delayed graft function: a paired kidney analysis. Transplantation. 2011;91(6):666-71.

10. Port FK, Bragg-Gresham JL, Metzger RA, Dykstra DM, Gillespie BW, Young EW, et al. Donor characteristics associated with reduced graft survival: an approach to expanding the pool of kidney donors. Transplantation. 2002;74(9):1281-6.

11.Mallon DH, Summers DM, Bradley JA, Pettigrew GJ. Defining delayed graft function after renal transplantation: simplest is best. Transplantation. 2013;96(10):885-9.

12.Levey AS, Bosch JP, Lewis JB, Greene T, Rogers N, Roth D. A more accurate method to estimate glomerular filtration rate from serum creatinine: a new prediction equation. Modification of Diet in Renal Disease Study Group. Ann Intern Med. 1999;130(6):461-70.
13.Bell ML, Fiero $\mathrm{M}$, Horton $\mathrm{NJ}$, Hsu $\mathrm{CH}$. Handling missing data in RCTs; a review of the top medical journals. BMC Med Res Methodol. 2014;14:118.

14. Haas M, Sis B, Racusen LC, Solez K, Glotz D, Colvin RB, et al. Banff 2013 meeting report: inclusion of c4d-negative antibodymediated rejection and antibody-associated arterial lesions. Am J Transplant. 2014;14(2):272-83.

15.Sandes Freitas TV S-J, HT. Disfunção inicial do enxerto. In: Jenner Cruz. Atualidades em Nefrologia 14. 1. São Paulo: SARVIER; 2016. p. 437-42.

16. Irish WD, Ilsley JN, Schnitzler MA, Feng S, Brennan DC. A risk prediction model for delayed graft function in the current era of deceased donor renal transplantation. Am J Transplant. 2010;10(10):2279-86.

17. Sandes-Freitas TV, Felipe CR, Aguiar WF, Cristelli MP, Tedesco-Silva H, Medina-Pestana JO. Prolonged Delayed Graft Function Is Associated with Inferior Patient and Kidney Allograft Survivals. PLoS One. 2015;10(12):e0144188. 